\title{
Sentinel-5 OGSE: large aperture sun simulator
}

J. Mendes-Lopes, J. Meyer, P. Borges, J. Pereira, R. Henriques, et al.

J. Mendes-Lopes, J. Meyer, P. Borges, J. Pereira, R. Henriques, J. Pimentao, D. Mesquita, "Sentinel-5 OGSE: large aperture sun simulator," Proc. SPIE 11852, International Conference on Space Optics - ICSO 2020, 118524E (11 June 2021); doi: 10.1117/12.2599653

SPIE Event: International Conference on Space Optics - ICSO 2021, 2021, Online Only 


\section{International Conference on Space Optics-ICSO 2020}

Virtual Conference

30 March-2 April 2021

Edited by Bruno Cugny, Zoran Sodnik, and Nikos Karafolas
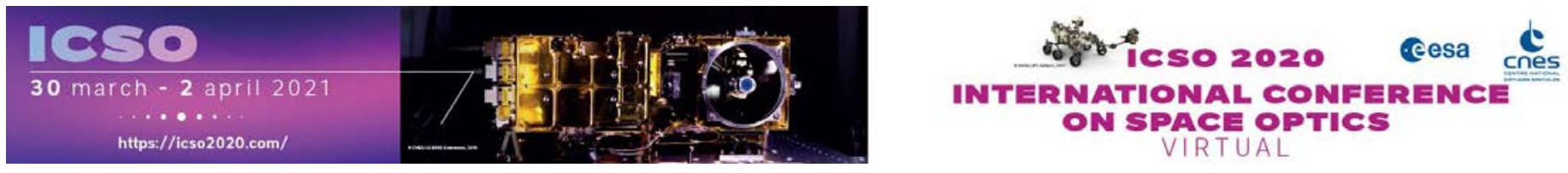

\section{Sentinel-5 OGSE: large aperture sun simulator}

\section{Cesa isopmeatians ecnes}




\title{
Sentinel-5 OGSE: Large Aperture Sun Simulator
}

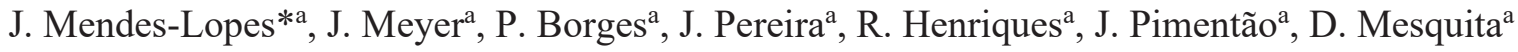 \\ ${ }^{a}$ Lusospace, Rua Sarmento Beires 31A, Lisboa, Portugal
}

\begin{abstract}
This paper reports on the design, development and testing of a sun simulator (SUSI) as an optical ground support equipment (OGSE) for the calibration and characterization (C\&C) of the Sentinel-5 instrument. The sun simulator is based on a Xenon continuous light source, a homogenizer and a telescope. A Xenon arc plasma source is significantly non-uniform, both spectrally and spatially. To meet the stringent spatial homogeneity requirements, a large homogenizer based on a kaleidoscope configuration was designed and manufactured. The proper collimation and large exit pupil are achieved through a mirror-based Offner telescope, avoiding large incidence angles, which would affect spatial uniformity and polarization.
\end{abstract}

SUSI covers a very wide spectral range, from 270 to $2385 \mathrm{~nm}$, with an irradiance of $80 \mathrm{~mW} / \mathrm{cm} 2$. SUSI provides an illuminated exit pupil with a very high spatial uniformity. The collimated beam of $0.318^{\circ}$ divergence illuminates an exit pupil $151 \mathrm{~mm}$ diameter with continuous light, and with a spatial non-uniformity better than 1\% (according to IEC 609049 definition) which is, to the best of the authors' knowledge, the highest level of spatial uniformity for large aperture sun simulators.

Keywords: OGSE, Sun simulator, Sentinel-5, calibration, characterization

*jmlopes@lusospace.com; phone +351 211165 020; lusospace.com

\section{INTRODUCTION}

The Sentinel-5 Mission [1] is dedicated to monitoring trace gas concentrations and aerosols in the atmosphere in a daily, near-real time basis. Data generated by the mission will be crucial and extremely useful for scientists and governments worldwide to assess climate and air-quality protocols. The earth observation is obtained by high-resolution spectrographs that are part of ESA's SENTINEL-5/UVNS Instrument onboard the MetOp - SG Satellite, in the context of the Copernicus Earth Observation Programme..

The Sentinel-5 instrument is an imaging push-broom passive UVVIS-NIR-SWIR spectrometer developed by Airbus Defense and Space $\mathrm{GmbH}$ as Prime Contractor under ESA contract [2]. The instrument consists of five spectrometers (UV-1, UV-2/Vis, NIR, SWIR-1 and SWIR-3), operating in spectral bands ranging from 270 to 2385 nm. Two coaligned reflective telescopes collect and transfer light to the spectrometers (US telescope feeds the UV-1, SWIR-1 and SWIR-3 spectrometers, while VN telescope provides the required photons to the UV-2/Vis and NIR spectrometer). Both telescope assemblies incorporate onboard calibration modules which, through a rotation wheel, switches the nominal Earth scene to different available calibration sources. Among other calibration sources, the calibration unit includes a diffuser and a Sun port, to use the Sun as calibration source for the spectrometers (Figure 1).

Lusospace is responsible for design, manufacturing, assembly and testing the OGSEs' units for Sentinel-5, to be used in both the Assembly, Integration and Testing (AIT) phase of UV1, UV2VIS and NIR spectrometers, and the Calibration and Characterization $(\mathrm{C} \& \mathrm{C})$ phase of the complete instrument. The on-ground calibration campaign, during the $\mathrm{C} \& \mathrm{C}$ phase, will include instrument measurements under flight representative conditions. These measurements will be carried out inside a dedicated thermal vacuum chamber.

Among the OGSE's developed by Lusospace, a fundamental one is the Sun Simulator (SUSI). SUSI will provide a perfectly collimated beam to characterize and calibrate the different instruments performance requirements, and also to retrieve calibration key parameters. The very stringent requirements of the instrument, both in dimensions of the beam and as in optical properties (spectral irradiance, spatial uniformity, aperture, among others), require a dedicated development of a large aperture Sun Simulator. 




Figure 1. Sentinel-5's VN telescope optical funcional block diagram. The Sun is used as a reference for the spectrometers' calibration

SUSI's performance was successfully verified and approved during the recent Factory Acceptance Test (FAT) campaign, and is currently waiting to be delivered for final testing at RAL Space, in the C\&C Test facilities. This paper presents the design and development of the instrument, details on the tests developed to evaluate its performance, and the experimental results of the test campaign.

\section{TECHNICAL DESCRIPTION OF SUN SIMULATOR}

\subsection{Requirements and characteristics}

The main goal of SUSI (Figure 2) is to provide the same features as the Sun for the calibration subsystem of the telescope, ensuring a full illumination, with similar spectral response, spatial uniformity, divergence and irradiance levels to the Sun. The main characteristics and requirements of the instrument are related with the large aperture $(>130 \mathrm{~mm})$, divergence level of the beam $\left(0.27^{\circ} \pm 0.05^{\circ}\right)$, spatial non-uniformity and irradiance level for a large spectral range (270 to $2385 \mathrm{~nm}$, covering the UV1, UV2, NIR, SWIR1 and SWIR3 ranges). The goal in creating a good solar simulator is to have these parameters match the sun's parameters as close as possible. For Sentinel-5, the requirements are especially stringent, as it is challenging to achieve high irradiance levels, while maintaining a divergence matching the Sun's, and very good spatial non-uniformity.

Table 1 summarizes the main characteristics of SUSI, based on experimental results from the test campaign.

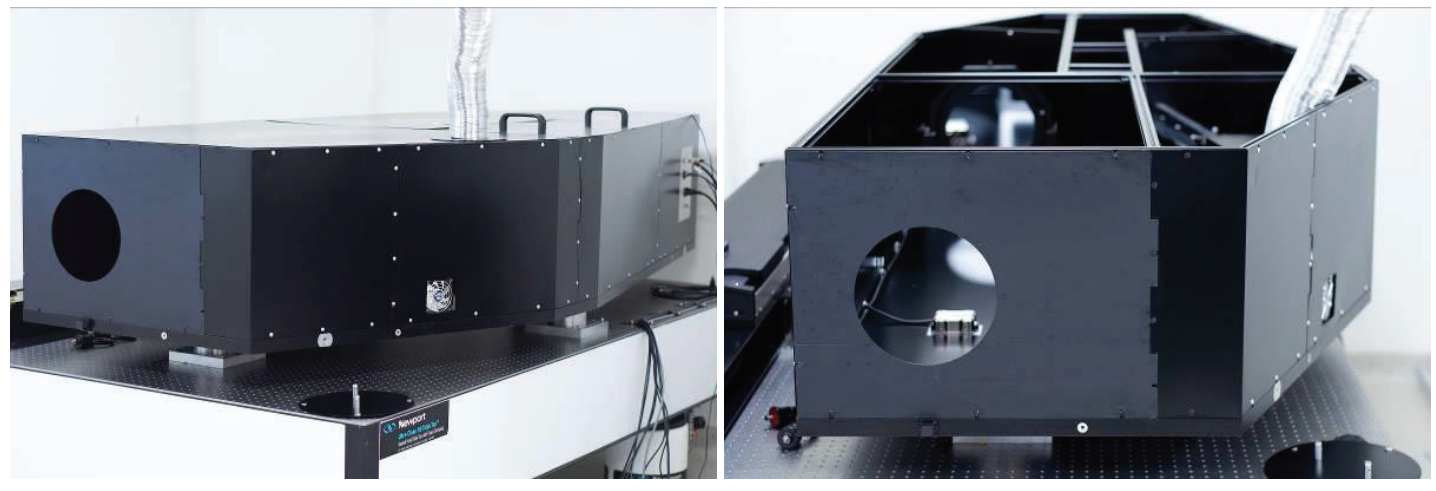

Figure 2. SUSI 
Table 1. Main characteristics of the Sun simulator (SUSI)

\begin{tabular}{|c|c|c|}
\hline \multicolumn{2}{|l|}{ Parameter } & \multirow{2}{*}{$\begin{array}{c}\text { Values } \\
\text { Xenon short arc lamp }\end{array}$} \\
\hline Light source & & \\
\hline \multirow{10}{*}{ Spectral irradiance, photons $\mathrm{cm}^{-2} \mathrm{~nm}^{-1}-\mathrm{s}^{-1}$} & UV1 & Min: $7.07 \mathrm{E}+12$ \\
\hline & $270-310 \mathrm{~nm}$ & Max: $9.09 \mathrm{E}+12$ \\
\hline & UV2 & Min: $1.29 \mathrm{E}+13$ \\
\hline & $300-500 \mathrm{~nm}$ & Max: $6.42 \mathrm{E}+13$ \\
\hline & NIR & Min: $2.75 \mathrm{E}+13$ \\
\hline & $685-773 \mathrm{~nm}$ & Max: $4.53 \mathrm{E}+13$ \\
\hline & SWIR1 & $1620 \mathrm{~nm}: 2.31 \mathrm{E}+13$ \\
\hline & $1590-1675 \mathrm{~nm}$ & $1653 \mathrm{~nm}: 1.63 \mathrm{E}+13$ \\
\hline & SWIR3 & $2318 \mathrm{~nm}: 1.15 \mathrm{E}+13$ \\
\hline & $2305-2385 \mathrm{~nm}$ & $2367 \mathrm{~nm}: 1.01 \mathrm{E}+13$ \\
\hline Beam diameter, mm & & 151 \\
\hline Half angle Beam divergence, degrees & & 0.318 \\
\hline$p p$ Spatial non-uniformity, $\%$ & $\begin{array}{c}\text { Inner pupil (60mm } \\
\text { diameter) } \\
\text { Wide pupil (151mm } \\
\text { diameter) }\end{array}$ & $\begin{array}{l}<1 \\
<5\end{array}$ \\
\hline Dimensions, $\mathrm{mm}$ & & $2430 \times 1040 \times 460$ \\
\hline
\end{tabular}

\section{Light source}

The optical design of the sun simulator was driven by the light source. For a continuous light flux, a short arc xenon lamp was selected, due to the good match with the spectral irradiance requirements. Although a Xenon arc lamp is widely used in sun simulators, for the stringent requirements of this instrument, it poses several challenges:

1) High thermal power - High optical power and low efficiency of the lamp leads to excessive heating. Sufficient clearance needs to be allowed between the optical components and the source, and the thermal stability needs to be accounted and analyzed in the design.

2) Time-dependent angular and spectral distribution - Xenon arc lamps produce their spectral emission creating a plasma between the cathode and anode. The light distribution is asymmetrical: more light emitted around the cathode than the anode. The spectral emissions have a spatial dependence - different wavelengths are emitted in different regions within the plasma. Moreover, the electrodes degrade during the lamp lifetime, which changes the angular and spectral distribution. These effects make the non-uniformity requirement even more challenging.

To quantify this effect, the spectral angular distribution of a xenon lamp was measured, using a spectrometer in the far field, in the 270-900nm spectral range. The lamp was measured in two different lifetimes, $75 \mathrm{~h}$ and $500 \mathrm{~h}$ (Figure 3). Results show both the asymmetry, the spectral dependence and time effect on the angular distribution. 

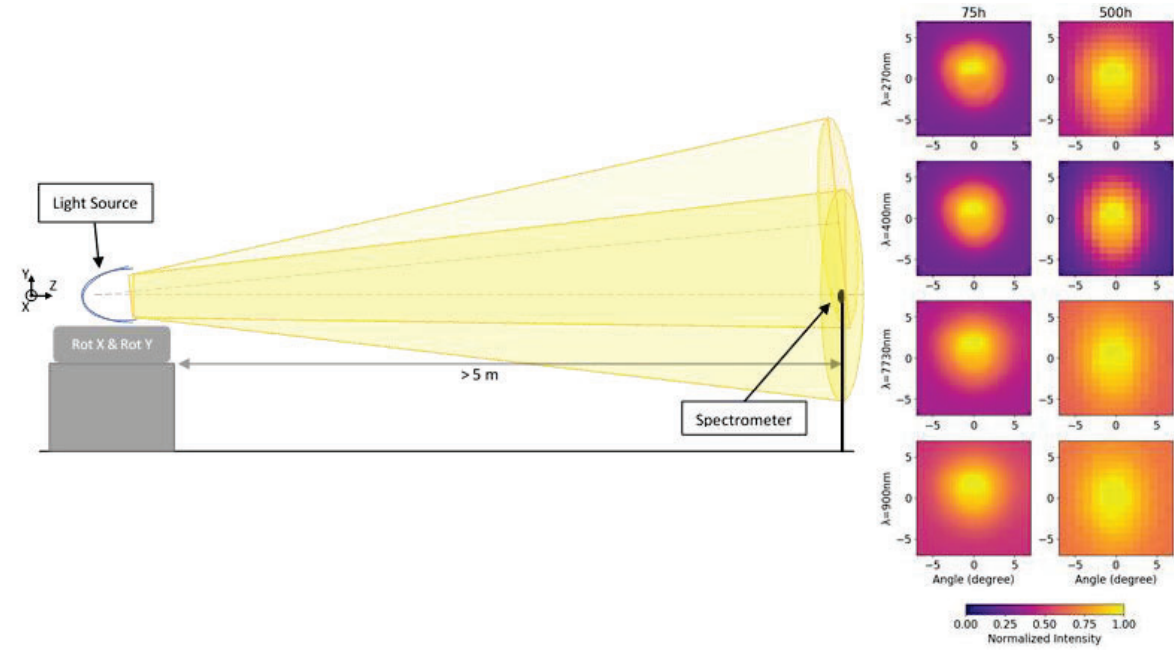

Figure 3. The far field angular distribution of a xenon arc lamp was measured in Lusospace's laboratory, in the spectral range $270-900 \mathrm{~nm}$, with the lamp in two different lifetimes, $75 \mathrm{~h}$ and $500 \mathrm{~h}$. The angular distribution is wavelength dependent, and is asymmetric in y axis for $75 \mathrm{~h}$, and it spreads for $500 \mathrm{~h}$.

3) High frequency intensity fluctuations - Xenon arc lamps have high frequency intensity fluctuations. Considering the purpose of the Sun simulator for the on-ground calibrations, active spectral monitoring of the light source is fundamental to account for these fluctuations.

\subsection{Optical design}

SUSI's optical design is composed of three parts: light source, homogenizer and telescope (Figure 4). A parabolic mirror collects and transmits the light from the light source into the homogenizer. The homogenizer consists on a customdesigned and made aluminum-mirrored kaleidoscope configuration, designed to achieve the intended spatial and spectral uniformity. The stringent spatial uniformity requirement demands special care in the design and optimization process. The measured spectral and time-dependent angular distribution of the Xenon lamp (Figure 3) was modeled in Zemax OpticStudio, and used in the overall design and optimization process. This modeling and respective optimization are crucial to ensure the performance throughout the lamp's lifetime, especially for the spectral irradiance and spatial uniformity.

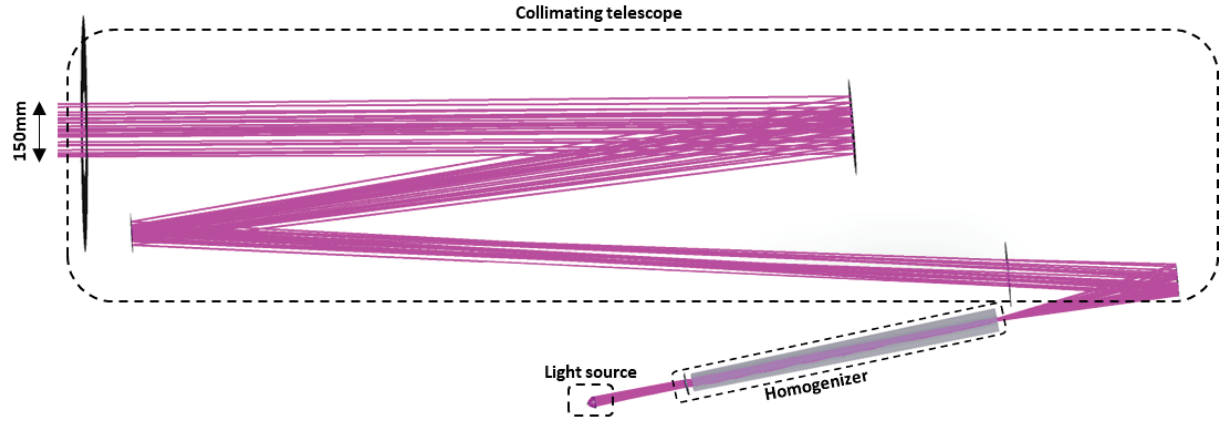

Figure 4. Optical layout of the sun simulator

The telescope is a mirror-based Offner telescope, with an incorporated field mask to ensure the divergence. All mirrors are coated with protected aluminum, and the design ensures the required collimation and magnification to achieve a large exit pupil $(>150 \mathrm{~mm})$. The telescope was designed to avoid large incidence angles, which would affect both spatial 
uniformity and polarization. The design was optimized such that the exit pupil is at $3178 \mathrm{~mm}$ from the simulator's envelope, although the distance can be easily changed and adapted.

\subsection{Structure and subsystems}

Figure 5 shows the CAD design of SUSI. The opto-mechanical support of the mirrors and field mask were designed with sufficient degrees of freedom for alignment and beam pointing. The baffle structure was selected to minimize straylight in the exit pupil, and highly anti-reflecting black coating is applied to straylight critical areas. This includes SUSI's front surface: SUSI's beam will be used through a window in the thermal vacuum chamber. The coating in the front surface prevents back reflections in the mirror to be reflected back to the vacuum chamber.

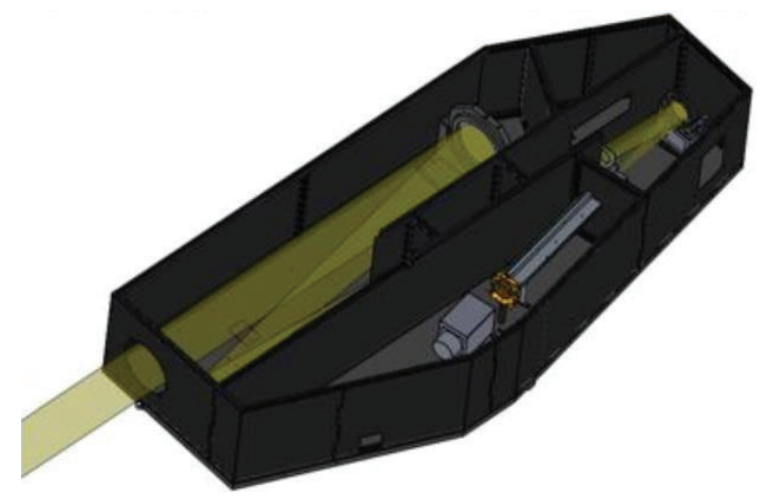

Figure 5. CAD design of SUSI

Thermal and structural analysis were made to ensure the performance of the system in ISO 8 cleanroom conditions (22 \pm $0.5^{\circ} \mathrm{C}$ ), including a pointing stability of 36 arcseconds.

SUSI's design includes a monitoring spectrometer, to perform active monitoring of the high frequency fluctuations of the light source, and a high speed shutter for the on ground calibration test campaign. It also includes an alignment cube and laser tracker balls for alignment purposes.

\section{TEST RESULTS}

The Factory Acceptance Test (FAT) of SUSI, was held in Lusospace's ISO 8 cleanroom during November and December 2020. Several tests were made, and several parameters were evaluated. For the purpose of the present paper, only the most relevant ones are presented:

- $\quad$ Spectral irradiance in the spectral regions of interest (UV1, UV2, NIR, SWIR1 and SWIR3);

- $\quad$ Beam divergence;

- Beam diameter;

- $\quad$ Spatial non-uniformity;

- Temporal stability of spatial non-uniformity. 


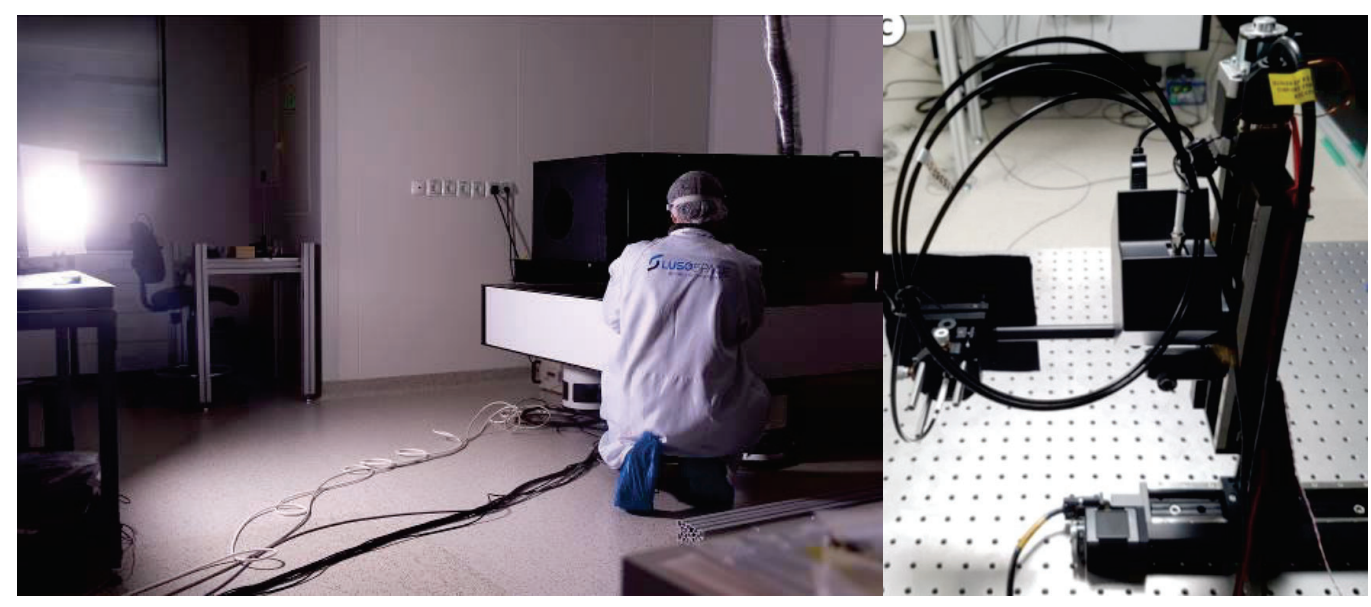

Figure 6. SUSI in the test campaign in Lusospace's ISO 8 cleanroom. On the left- SUSI's optical beam is impinging into a diffuser at $3178 \mathrm{~mm}$. On the right - A fiber-tip spectrometer on a 2D stage was used for the spatial homogeneity measurements

\subsection{Spectral irradiance}

The spectral irradiance was measured using a calibrated spectrometer for UV1, UV2 and NIR, and with IR photodiode and filters for SWIR1 (1620 and 1653nm) and SWIR3 (2318 and 2367nm). The results showed full compliance with the requirements. Table 2 shows the irradiance full each spectral band, and Figure 7 shows the spectrum for the range 270 to $773 \mathrm{~nm}$.

Table 2. Irradiance measurement results (photons.cm ${ }^{-2} \mathrm{~nm}^{-1} \mathrm{~s}$
\begin{tabular}{|c|c|}
\hline SPECTRAL RANGE & RESULT \\
\hline UV1 & Min: $7.07 \mathrm{E}+12$ \\
270-310nm & Max: $9.09 \mathrm{E}+12$ \\
\hline UV2 & Min: $1.29 \mathrm{E}+13$ \\
300-500nm & Max: $6.42 \mathrm{E}+13$ \\
\hline NIR & Min: $2.75 \mathrm{E}+13$ \\
$685-773 \mathrm{~nm}$ & Max: $4.53 \mathrm{E}+13$ \\
\hline SWIR1 & $1620 \mathrm{~nm}: 2.31 \mathrm{E}+13$ \\
$1590-1675 \mathrm{~nm}$ & $1653 \mathrm{~nm}: 1.63 \mathrm{E}+13$ \\
\hline SWIR3 & $2318 \mathrm{nm:}: 1.15 \mathrm{E}+13$ \\
$2305-2385 \mathrm{~nm}$ & $2367 \mathrm{~nm}: 1.01 \mathrm{E}+13$ \\
\hline
\end{tabular}

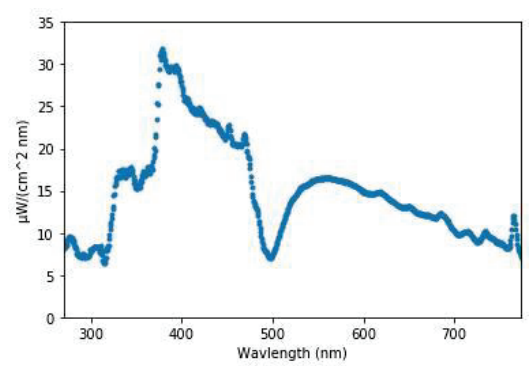

Figure 7. Spectral response from 270 to $773 \mathrm{~nm}$

\subsection{Beam divergence}

The beam divergence was measured considering the optical design of the system. Considering that the divergence is defined by a field mask, a theodolite, focused into infinity, was placed in the exit pupil position, and pointed in the opposite direction of the beam. With the focused image of the field mask in the theodolite, its angular aperture was 
measured. The divergence half-angle was measured to be $\pm 0.318^{\circ}$, precisely the design value (which was maximized to improve the spectral irradiance).

\subsection{Spatial non-uniformity and beam diameter}

The beam diameter was measured to be $151 \mathrm{~mm}$. For such a large beam diameter, and for the intended measurement accuracy $(0.05 \%)$, a dedicated test setup was developed. A fiber tip spectrometer was setup on top of a $2 \mathrm{D}$ stage, to perform a $2 \mathrm{D}$ scan of the entire exit pupil, with $3 \times 3 \mathrm{~mm}$ pixels (Figure 6). During scanning, SUSI's shutter and monitoring spectrometer were used to normalize the data with dark current and source intensity fluctuations measurements, respectively.
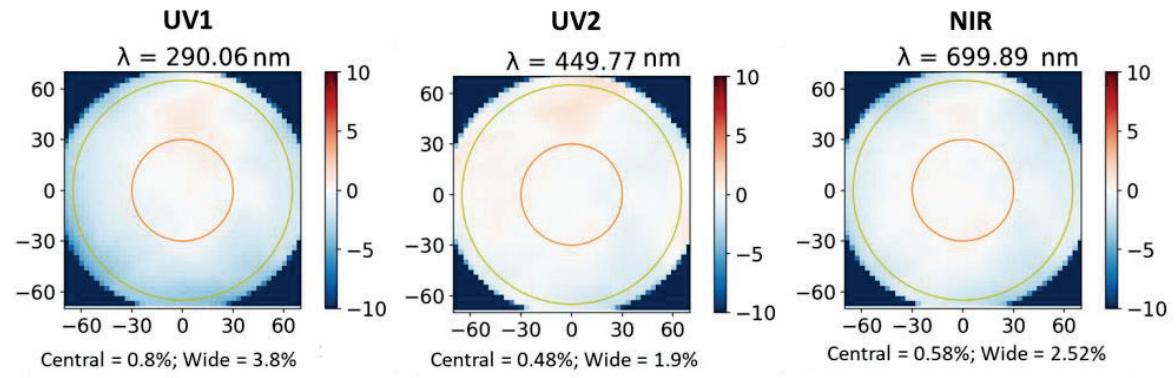

SWIR1

SWIR3
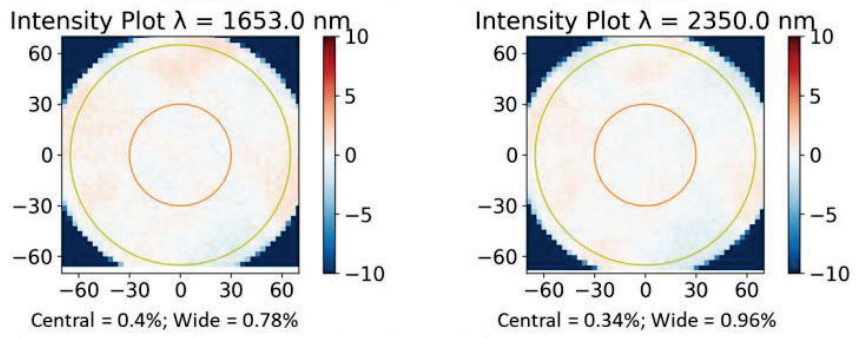

Figure 8. Measured irradiance patterns for representative wavelengths in each spectral band, in percentage. The values below each plot are the $p p$ spatial non-uniformity for the central inner exit pupil (60 mm diameter) and wide exit pupil

$(151 \mathrm{~mm})$

The reported spatial non-uniformity values are peak-to-peak, according to IEC 60904-9 definition. Figure 8 presents the measured irradiance patterns for representative wavelengths of each spectral band. The $p p$ spatial non-uniformity values are presented below each plot: Central are the values for a $60 \mathrm{~mm}$ diameter region of the exit pupil, while Wide are the values for a $151 \mathrm{~mm}$ diameter exit pupil. The results show that the irradiance patterns are extremely homogeneous. Across all spectral regions of interest, the spatial non-uniformity is below 1\% for the central exit pupil; for the wide exit pupil, it is below 5\%. The reported values are, to the best of the authors' knowledge, the highest value of spatial homogeneity for large aperture sun simulators.

\subsection{Temporal stability of spatial homogeneity}

The fact that the angular distribution of the Xenon lamp changes over the lamp's lifetime might have an impact on the spatial homogeneity. As such, the spatial non-uniformity was measured 7 times, spread across 240 hours, with the lamp always turned on (approximately the estimated time required for Sentinel-5 test campaign), in UV1, UV2 and NIR spectral ranges (Figure 9). The results have confirmed the stability of the spatial homogeneity, maximum variation occurring in NIR, of $0.15 \%$. 


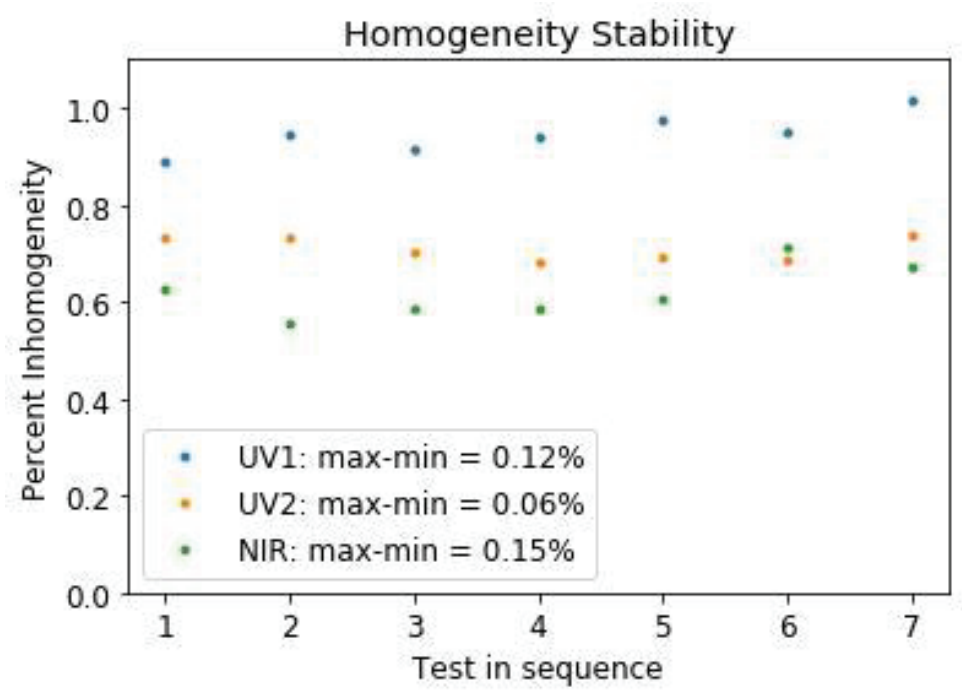

Figure 9. Homogeneity stability. With the Xenon lamp always turned on, the spatial non-uniformity was measured 7 times spread across 240 hours.

\section{CONCLUSION}

The paper described the characteristics, final design and development of the Sun Simulator (SUSI) developed as part of the OGSEs for Sentinel-5, to be used for the on-ground calibration of the instrument. The results of the Factory Acceptance Testing (FAT) campaign are reported in the paper, and confirm the excellent performance of the OGSE. SUSI provides a $151 \mathrm{~mm}$-diameter beam, with a half angle divergence of $0.318^{\circ}$. The central diameter of the beam $(60 \mathrm{~mm})$ has a spatial non-uniformity better than $1 \%$, and the wide diameter of the beam (151mm) has a spatial nonuniformity better than $5 \%$. The reported values are, to the best of the authors' knowledge, the highest value of spatial homogeneity for large aperture sun simulators.

SUSI's performance was successfully verified and approved during the recent Factory Acceptance Test (FAT) campaign, and is currently waiting to be delivered for final testing at RAL Space, in the C\&C Test facilities.

\section{REFERENCES}

[1] J. Irizar, M. Melfa, P. Bartsch, J. Koehle, S. Weiss, R. Greinacher, M. Erdmann, V. Kirschner, A. Perez Albinana, D. Martin, "Sentinel-5/UVNS", Proceedings of SPIE: International Conference on Space Optics. Vol. 11180 (2018)

[2] “Sentinel-5", ESA SP-1336, January 2020 